Conclusion: Our study showed that there was no correlation between the clinical condition of patients and their follow-up imaging in the context of PVO. Clinical and biological evaluation seems sufficient to determine whether or not the patient is cured. Many images are made during the follow-up with a questionable cost/effectiveness ratio. A standard radiograph may be sufficient to provide a basic structural condition at the end of antibiotic therapy.

Disclosure of Interests: None declared

DOI: 10.1136/annrheumdis-2019-eular.4842

\section{SAT0463 BRUCELLAR SPONDYLODISCITIS: CLINICAL, RADIOLOGICAL AND THERAPEUTIC FEATURES}

Aicha Ben Tekaya ${ }^{1}$, Khaoula Zouaoui ${ }^{1}$, Ines Mahmoud ${ }^{1}$, Olfa Saidane ${ }^{1}$, Mohamed Ben Hammamia ${ }^{2}$, Rawdha Tekaya ${ }^{1}$, Leila Abdelmoula $1 .{ }^{1}$ Charles Nicolle Hospital, Tunis, Tunisia; ${ }^{2}$ la rabta hospital, Tunis, Tunisia

Background: Brucellosis is an endemic disease around the Mediterranean and especially in Tunisia and Brucellar spondylodiscitis is the most common osteoarticular localization.

Objectives: The aim of our study is to study the,clinical, radiological and therapeutic characteristics of Brucellar spondylodiscitis.

Methods: This is a retrospective descriptive study, conducted over 20 years (1999-2019) at a Rheumatology Department. We collected cases of Brucellar spondylodiscitis. We studied the clinical, radiological features and therapeutics outcomes.

Results: We included 23 patients, 15 men and 8 women, with a mean age of 53.21 years [31,79].

Contact with livestock or consumption of raw milk was noted in 16 cases. The diagnosis time was, on average, 3.8 months [1,9]. Spine pain was present in all cases, with lumbar seat in 16 cases and was inflammatory in 20 cases. At the examination, 19 patients had a limitation of spinal mobility and 4 had neurological abnormalities. A motor deficit with a horsetail syndrome was objectified in one case. We noted a biological inflammatory syndrome in 19 cases. Wright's serology was positive in 21 cases. Standard radiographs showed disc narrowing in 10 cases. 21 patients had spinal magnetic resonance imaging showing the abnormalities of the disc and adjacent vertebrae. We found abscess in four patients and epiduritis associated with the abscess in six patients. MRI showed spinal compression in 2 patients. Disco-vertebral biopsy was performed in 11 cases and helped to make the diagnosis in 3 cases. The patients had received antibiotic therapy with a combination of doxycylin and rifampicin with a mean total duration of 4.9 months [2.12]. Evolution was favorable in 19 cases. Complications were mainly neurological $(n=3)$ or related to the toxicity of the treatment $(n=3)$.

Conclusion: Brucellar spondylodiscitis can be serious because of the neurological complications that it can cause. At the slightest diagnostic doubt, we should perform an MRI. And antibiotic treatment must be urgent and well-adapted with careful monitoring of patients.

Disclosure of Interests: None declared

DOI: 10.1136/annrheumdis-2019-eular.6229

\section{SAT0464 HEPATITIC SAFETY OF ANTI-TUBERCULOUS TREATMENT IN SPONDYLODISCITIS}

Aicha Ben Tekaya ${ }^{1}$, Khaoula Zouaoui ${ }^{1}$, Rawdha Tekaya ${ }^{1}$, Olfa Saidane ${ }^{1}$, Mohamed Ben Hammamia ${ }^{2}$, Ines Mahmoud ${ }^{1}$, Leila Abdelmoula ${ }^{1,1} .{ }^{1}$ Charles Nicolle Hospital, Tunis, Tunisia; ${ }^{2}$ la rabta hospital, Tunis, Tunisia

Background: Tunisia is considered as a country with high tuberculosis endemicity. The anti-tuberculous treatment is quite long and binding and requires close hepatic monitoring.

Objectives: The purpose of this study was to highlight the hepatitic safety of anti-tuberculous treatment in tuberculous spondylodiscitis.

Methods: This is a retrospective descriptive study, over 20 years (19992019) collating cases of tuberculous spondylodiscitis in a rheumatology department. We studied the epidemiological, clinical, radiological and therapeutic aspects.

Results: Our study included 62 patients, 35 women and 27 men. Mean age was 56 years [16-86]. The diagnosis delay averaged 5.59 months [0.23-24]. Tuberculous contact was noted in $11.3 \%$ of the cases. Neurological abnormalities were noted in $16,1 \%$ of cases with spine compression in $3,22 \%$. The tuberculin skin test was positive in 29 cases and the Koch bacillus investigations in the sputum and the urine were positive in only 3 patients. Magnetic resonance imaging was performed in $71 \%$ of the patients, and mainly showed images of disc destruction with images of abscess, epiduritis and epidural extension. Infectious spondylodiscitis affected the lumbar spine in $66.1 \%$ of the cases, the dorsal spine in $14.51 \%$ of the cases and the cervical spine in $6.55 \%$ of the cases. It was bi-staged in $19.35 \%$ of the cases and bifocal in $17.74 \%$ of the cases.

Disco-vertebral biopsy was performed for $72.5 \%$ of patients and helped to make the diagnosis in $33.87 \%$ of the cases. All patients have received anti-tuberculosis treatment based on rifampicin, pyrazinamide, ethambuto and Isoniazid for an average duration of 2.8 months. Following the initial 4-drug regimen, most patients continued to receive a two-drug regimen with RMP and INH for a mean duration of nine months. Hepatotoxicity was seen in 13\%: $11.4 \%$ of the patients had a history of cholestasis due to TB treatment, Only $2 \%$ of the patients had cytolysis. We nedded then to modify the treatment in $3,22 \%$, and switch to triple anti-TB therapy based on Isoniazid, Rifampicin and ethambutol with a favorable evolution. No cases of hepatic insufficiency were noted.

Conclusion: In our study, we note 8 cases of hepatotoxicity. The diagno sis of tuberculous spondylodiscitis requires urgent treatment with antituberculosis antibiotics. However, it should be kept in mind that this treatment can lead to severe and life-threatening hepatotoxicity. Thus a rigorous monitoring of the treatment will be required.

Disclosure of Interests: None declared

DOI: 10.1136/annrheumdis-2019-eular.7460

\section{SAT0465 VALUE OF SERUM PROCALCITONIN FOR THE DIAGNOSIS OF BACTERIAL SEPTIC ARTHRITIS IN DAILY PRACTICE IN RHEUMATOLOGY}

Mickaël Chouk ${ }^{1}$, Frank Verhoeven ${ }^{1}$, Maxime Sondag ${ }^{1}$, Xavier Guillot $^{2}$, Clément Prati ${ }^{1}$, Daniel Wendling ${ }^{1} .{ }^{1}$ Centre hospitalier régional universitaire de Besançon, Besançon, France; ${ }^{2}$ Hospital Center Félix Guyon, Saint-Denis, Reunion Island

Background: Septic arthritis is a diagnostic and therapeutic emergency because of a high morbidity and mortality. Nevertheless, the etiologic diagnosis is often difficult.

Objectives: The aim of our study was to determine if serum procalcitonin was a discriminatory biomarker in case of arthritis of undetermined etiology.

Methods: Patients were separated in 5 groups: gouty arthritis, calcium pyrophosphate deposition arthritis, osteoarthritis or post-traumatic arthritis ("mechanical" arthritis), chronic inflammatory rheumatic arthritis, and septic arthritis. Levels of serum with blood cells, C-Reactive Protein and procalcitonin were measured.

Results: 98 patients were included: 18 in the "gout" group, 26 in the "calcium pyrophosphate deposition arthritis" group, 16 in the "mechanical" group, 18 in the "chronic inflammatory rheumatic" group and 20 in the "sepsis" group. The area under the receiver operating characteristic curve of with blood cells, C-Reactive Protein and procalcitonin levels to diagnose a septic arthritis were 0.69 (IC95\% $0.55-0.83$ ), 0.82 (IC95\% 0.73 0.91 ), and 0.87 (IC95\% 0.76-0.98) respectively. For a cut-off of $0.5 \mathrm{ng} /$ $\mathrm{ml}$, procalcitonin sensitivity, specificity, positive predictive value, negative predictive value, positive likelihood ratio and negative likelihood ratio were $65 \%, 91 \%, 65 \%, 91 \%, 7.2$ and 0.4 , respectively. Serum C-Reactive Protein and procalcitonin levels were correlated, were not different in "sepsis" or "gout" groups and were higher in non-septic arthritis with poly-arthritis than with mono-arthritis $(p<0.05)$.

Conclusion: Serum procalcitonin is a useful biomarker in arthritis manage ment with diagnosis performances higher than those of other biomarkers (with blood cells, C-Reactive Protein).

Disclosure of Interests: None declared

DOI: 10.1136/annrheumdis-2019-eular.2686

\section{SAT0466 PNEUMOCOCCAL CELLULITIS AND FASCIITIS IN SYSTEMIC LUPUS ERYTHEMATOSUS: A SYSTEMATIC REVIEW}

Martín Greco ${ }^{1}$, Iñigo Rua-Figueroa ${ }^{1}$, Antonio Naranjo ${ }^{1}$, Sabrina Ghiglione ${ }^{2}$, Maria Celia Erausquin ${ }^{1}$, Juan Carlos Quevedo-Abeledo ${ }^{1}$, Carlos Rodriguez-Lozano ${ }^{1}$, Cristina Almeida ${ }^{1}$, Paola León ${ }^{1}$, Estíbaliz Loza ${ }^{3} .{ }^{1}$ Hospital Universitario de Gran Canaria Dr. Negrín., Las Palmas, Spain; ${ }^{2}$ Complejo Hospitalario Universitario Insular Materno Infantil - CHUIMI, Las Palmas, Spain; ${ }^{3}$ Instituto de Salud Musculoesquelética Inmusc, Madrid, Spain

Background: Streptococcus pneumoniae (SPN) is an encapsulated gram positive bacterium that can be found in the nasopharynx as part of the normal flora. However, is the most common cause of community-acquired pneumonia in adults and can also cause invasive diseases such as bacteremia, meningitis, and otitis media. Pneumococcal cellulitis and fasciitis 